\title{
Successful Use of Single-Dose Rituximab for the Maintenance of Remission in a Patient with Steroid-Resistant Nephrotic Syndrome
}

\author{
Noritomo Kurosu, Hidekazu Sugiura, Chihiro Iwasaki, Yukari Asamiya, Chiari Kojima, \\ Takahito Moriyama, Mitsuyo Itabashi, Misao Tsukada, Takashi Takei, Tetsuya Ogawa, \\ Takumi Yoshida, Keiko Uchida, Ken Tsuchiya and Kosaku Nitta
}

\begin{abstract}
We present the case of a 23-year-old man with steroid-resistant nephrotic syndrome due to minimal change disease who was treated with rituximab. The patient was resistant to conventional therapy. We therefore treated him with a single dose of rituximab $\left(375 \mathrm{mg} / \mathrm{m}^{2}\right)$. One month after the administration of rituximab, complete remission was achieved. However, six months later, the patient was administered a second dose of rituximab as the peripheral $\mathrm{B}$ cell counts began to recover. Thereafter, at present, that is, one year after the first rituximab administration, complete remission has been maintained. We conclude that rituximab may be an effective treatment agent for resistant nephrotic syndrome and the peripheral B cell count may be a useful marker in such patients for preventing disease relapse.
\end{abstract}

Key words: steroid-resistant nephritic syndrome, minimal change nephritic syndrome, rituximab, peripheral B-cell

(Inter Med 48: 1901-1904, 2009)

(DOI: 10.2169/internalmedicine.48.2435)

\section{Introduction}

Minimal-change disease (MCD) is a common cause of steroid-resistant nephrotic syndrome (SRNS), and no standard therapies have been firmly established yet. The pathophysiology of minimal-change nephrotic syndrome (MCNS) remains poorly understood (1). Recently, it was reported that rituximab (an anti-CD20 monoclonal antibody) has been successfully used to treat patients with several renal diseases (2).

We report a case of SRNS due to MCD in which remission was achieved following treatment with rituximab. When the peripheral $\mathrm{B}$ cell counts began to recover, he received a second dose of rituximab, thereby relapse was prevented. Complete remission has been maintained for one year. Our experience with this patient suggests that B cells may also be involved in the pathophysiology of MCNS.

\section{Case Report}

A man patient of Japanese origin was diagnosed as having idiopathic nephrotic syndrome (INS) when he was nine years old (1993). Until that age, his medical history had been unremarkable. A kidney biopsy at the time of diagnosis of the nephrotic syndrome showed minimal-change disease. Successful treatment was accomplished with standard prednisolone treatment. After reduction of the steroid dose, the patient experienced his first relapse in 1994. He responded again to steroids, but thereafter developed frequent relapses and became steroid dependent; he was thus labeled as having steroid-dependent nephrotic syndrome (SDNS). This led to the step-by-step use of the currently available medications for patients with SDNS in addition to steroid therapy, including cyclosporine (CyA) at the time of the second relapse in 1994. Only partial remission could be in- 


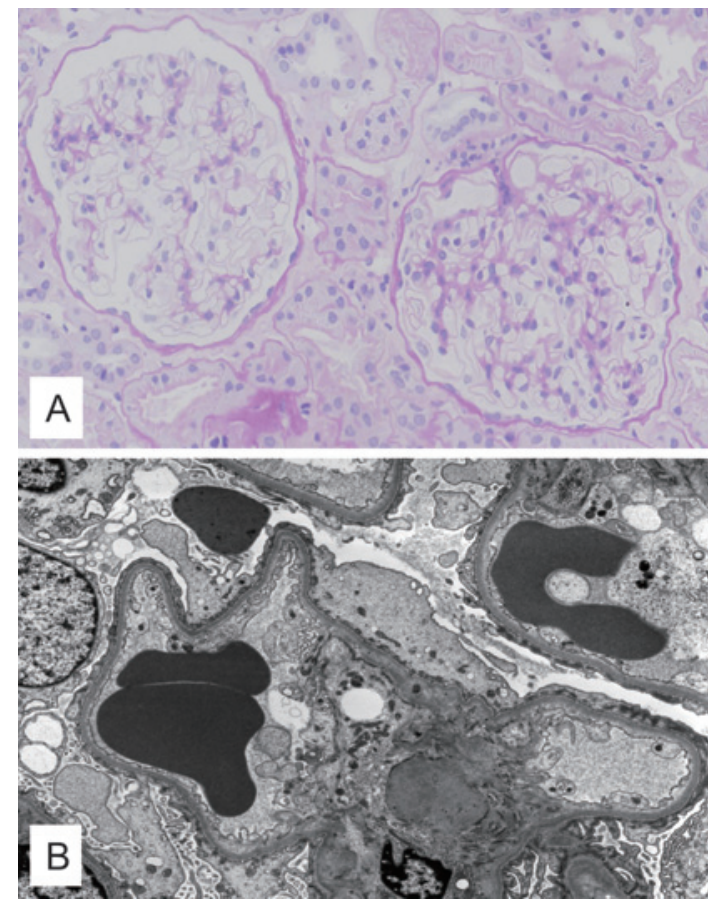

Figure 1. Renal biopsy findings, A) Light microscopy showed minor abnormality (Periodic acid-Schiff $\times 400$ ). B) Electro-micrography showed widespread glomerular foot process effacement $(\times 3,500)$.

duced by the combined therapies, and the prednisolone dosage remained at $20 \mathrm{mg} /$ day.

Over the course of the subsequent 20 or so relapses, he became progressively more resistant to steroids and came to be labeled as a case of SRNS. Therefore, a second kidney biopsy was performed in 2001. Pathological examination revealed that all of the 15 glomeruli examined were of minor abnormality. There was no mesangial proliferation and no increase of mesangial matrices. No significant changes were observed in the tubules, interstitium, and vasculature. Immunofluorescence studies did not reveal any immune deposits. Electron-microscopic studies demonstrated widespread glomerular foot process effacement (Fig. 1). The pathological diagnosis was again $\mathrm{MCD}$, as after the first biopsy. Although mizoribine was added to the steroids and cyclosporine, it did not appear to exert any beneficial effect.

The patient was admitted to the Department of Medicine at Tokyo Women's Medical University in May 2007 because of SRNS. On admission, his urinalysis revealed massive proteinuria $(5.0 \mathrm{~g} /$ day $)$ without hematuria. Serum total protein, albumin and total cholesterol were $3.6 \mathrm{~g} / \mathrm{dL}, 1.4 \mathrm{~g} / \mathrm{dL}$ and $603 \mathrm{mg} / \mathrm{dL}$, respectively. His renal function was normal. Determination of the proteinuria selectivity index based on Cameron's selectivity index in terms of the IgG-transferring clearance ratio was 0.1 . He was started on combination therapy consisting of semi-pulse mPSL, mycophenolate mofetil (MMF), and low-density lipoprotein-apheresis (LDL-A), but despite these treatments, remission was not achieved (Fig. 2).

As a last resort, we decided to treat him with rituximab, even though it is classically used only to treat patients with B cell-dependent autoimmune diseases. We administered single-dose rituximab (a single infusion of $375 \mathrm{mg} / \mathrm{m}^{2}: 500$ $\mathrm{mg}$ ). Complete remission occurred one month later, when CD19/20-positive lymphocytes were no longer detected in the blood. Complete remission was maintained for six months despite discontinuation of the steroid and CyA. At six months after the first rituximab dose, when the peripheral B cell counts began to increase (CD19: $11.5 \%$ of total lymphocytes, CD20: $9.8 \%$ of them), a second dose of rituximab was administered (a single infusion of $375 \mathrm{mg} / \mathrm{m}^{2}: 500$ $\mathrm{mg}$ ). Following this dose, the peripheral B cell counts declined rapidly. No rapid differences were noted in the CD4/ CD8 ratios determined before and after the rituximab treatment, but there was an increase. To date, one year after the first dose of rituximab, complete remission has been maintained. No adverse events have been observed during the follow-up period.

\section{Discussion}

The rationale for using immunosuppressants in the treatment of MCNS is based on the pathophysiology of MCNS, which is considered to be an immune disorder in which a permeability factor, possibly produced by $\mathrm{T}$ lymphocytes, induces albuminuria (1). Although MCNS is not antibodymediated disease, B lymphocytes play an important role as immunoregulatory cells by both antigen presentation and cytokine release.

Although steroids remain the cornerstone of treatment, various other immunosuppressants have proved effective in preventing relapses and decreasing steroid dependency (3). CyA administration might play a role in reducing the frequency and severity of steroid toxicity. However, several reports have indicated that protracted use of CyA is also sometimes associated with chronic renal injury and hypertension, and development of malignant tumors has been reported in some patients with INS treated with CyA (4). Previous reports have shown that mizoribine therapy appears to be effective for reducing the CsA exposure level as well as decreasing the frequency of relapses in patients with SDNS and SRNS who are also CsA-dependent (5). The rapid improvement of hypercholesterolemia in response to LDL-A therapy suggests a new approach to achieving a high rate of improvement in the severity of SRNS (6). One previous report has suggested that MMF therapy might be effective in SRNS patients in whom other immunosuppressant therapy has failed (7). However, neither mizoribine, LDL-A or MMF had any beneficial effects in the present patient.

We then decided to administer rituximab. Recent studies have reported that rituximab therapy may be used successfully in patients with renal disease (2). In a previous study, children with INS responded well to rituximab therapy (8). The case of a 23-year-old man who suffered from 12 relapses of MCNS was reported recently, in whom long-term remission was achieved only with a standard dosing regimen 


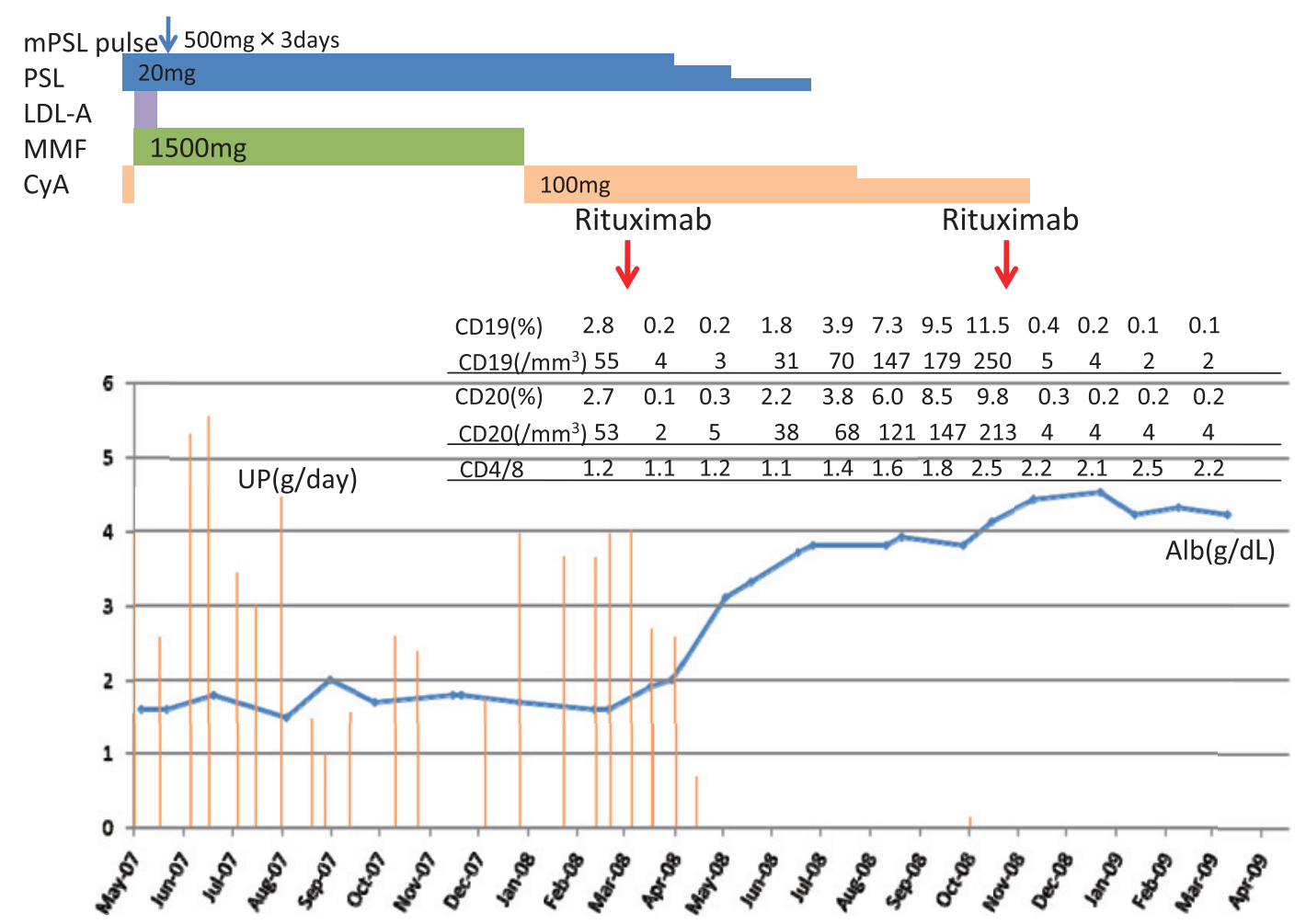

Figure 2. Clinical course of the patient at our hospital.

of rituximab (375 mg/m² every week for 4 weeks) after failure of successive treatments with all other currently available medications for MCNS (9). In most studies, rituximab has generally been prescribed at the dose for which it was developed and licensed for the treatment of B cell nonHodgkin's lymphoma (NHL), $375 \mathrm{mg} / \mathrm{m}^{2}$ weekly for 4 consecutive weeks (10). However, the tumor burden in lymphomas is often high whereas in ITP and other autoimmune hematologic disorders the $\mathrm{B}$ cell mass is presumably normal. We used single-dose (500 $\mathrm{mg}$ fixed dose) rituximab to treat the present patient and the single dose sufficient for longterm CD20 expressing B cell depletion.

Rituximab targets CD20-expressing B cells, but the drug may also affect $\mathrm{T}$ cell function by inducing $\mathrm{B}$ cell depletion (11). In MCNS patients, rituximab may alter production by the $\mathrm{T}$ cells, of the supposed permeability factors, whose cytokine nature is still under debate. In our patient, The CD4/8 ratios increased after CyA and PSL were reduced and discontinued. CyA stems from the inhibition of nuclear factor of activated $\mathrm{T}$ cells signaling in $\mathrm{T}$ cells. CyA, PSL and rituximab combined therapy might be effective for immunosuppressive action.

Recovery of the peripheral B cell counts began to be observed in our patient by six months after the first infusion in our patient. In recent report, the efficacy of rituximab was assessed in 22 patients with SDNS and SRNS. No relapse of proteinuria was recorded during the $\mathrm{B}$ cell depletion period. Relapse occurred between 54 (3\% of total lymphocyte

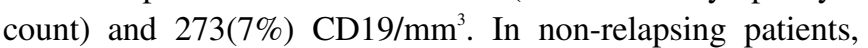
systemic rituximab re-infusions were performed between 10 $(1 \%)$ and $270(7 \%) \mathrm{CD} 19 / \mathrm{mm}^{3}$ in accordance with the thera- peutic guidelines (12). However, it was debatable whether or not proteinuria had relation to CD19/CD20. He was therefore administered a second dose of rituximab, because we hypothesized that the elimination of CD19/CD20-positive lymphocytes may be necessary to prevent disease relapse in this case. B-lymphocytes may play an important role in the pathogenesis of MCNS and the peripheral B cell count may serve as a useful marker in the management of SRNS secondary to MCD.

We conclude that B-lymphocytes may play an important role in the pathogenesis of MCNS. Rituximab may serve as a useful therapeutic agent for SRNS due to MCD. Our experience suggests that the peripheral blood count of CD20/CD 19-positive lymphocytes may serve as a useful marker for preventing disease relapse and may indicate the need for repeated use of rituximab in patients with SRNS. This is the first case report of the efficacy of single-dose rituximab therapy administered twice in maintaining remission in a patient with SRNS.

\section{Acknowledgement}

The authors thank Chugai Pharmaceutical Co., Ltd. for their technical support and Mr. Shigeru Horita for histological assessment. 


\section{References}

1. Cunard R, Kelly CJ. T cells and minimal change disease. J Am Soc Nephrol 13: 1409-1411, 2002.

2. Salama AD, Pusey CD. Drug insight: rituximab in renal disease and transplantation. Nat Clin Pract Nephrol 2: 221-230, 2006.

3. Inoue $\mathrm{Y}$, Iijima $\mathrm{K}$, Nakamura $\mathrm{H}$, Yoshikawa $\mathrm{N}$. Two year cyclosporin treatment in children with steroid-dependent nephrotic syndrome. Pediatr Nephrol 13: 33-38, 1999.

4. Collaborative Study Group of Sandimmun In Nephrotic Syndrome. Safety and tolerability of cyclosporine A (Sandimmun) in idiopathic nephrotic syndrome. Clin Nephrol 35: S48-S60, 1991.

5. Kawasaki Y, Hosoya M, Kobayashi S, et al. Oral mizoribine pulse therapy for patients with steroid-resistant and frequently relapsing steroid-dependent nephrotic syndrome. Nephrol Dial Transplant 20: 2243-2237, 2005.

6. Muso E, Mune M, Fujii Y, et al; Kansai-FGS-Apheresis Treatment (K-FLAT) Study Group. Low density lipoprotein apheresis therapy for steroid-resistant nephrotic syndrome. Kidney Int Suppl $71:$ S 122-S125, 1999.

7. Pesavento TE, Bay WH, Agarwal G, Hernandez RA Jr, Hebert LA. Mycophenolate therapy in frequently relapsing minimal change disease that has failed cyclophosphamide therapy. Am J Kidney Dis 43: e3-e6, 2004.

8. Benz K, Dotsch J, Rascher W, Stachel D. Change of the course of steroid-dependent nephrotic syndrome after rituximab therapy. Pediatr Nephrol 19: 794-797, 2004.

9. Francois H, Daugas E, Bensman A, Ronco P. Unexpected efficacy of rituximab in multirelapsing minimal change nephrotic syndrome in the adult: first case report and pathophysiological considerations. Am J Kidney Dis 49: 158-161, 2007.

10. Maloney DG, Grillo-Lopez AJ, White CA, et al. IDEC-C2B8 (Rituximab) anti-CD20 monoclonal antibody therapy in patients with relapsed low-grade non-Hodgkin's lymphoma. Blood 90: 21882195, 1997.

11. Eisenberg R, Looney RJ. The therapeutic potential of anti-CD20: "What do B cells do?". Clin Immunol 117: 207-213, 2005.

12. Guigonis V, Dalloccchio A, Baudouin V, et al. Rituximab treatment for severe steroid- or cyclosporine-dependent nephritic syndrome: a multicentric series of 22 cases. Pediatr Nephrol 23: 1269-1279, 2008.

(C) 2009 The Japanese Society of Internal Medicine http://www.naika.or.jp/imindex.html 